Annals of Pure and Applied Mathematics

Vol. 16, No. 2, 2018, 323-335

ISSN: 2279-087X (P), 2279-0888(online)

Published on 22 February 2018

www.researchmathsci.org

DOI: http://dx.doi.org/10.22457/apam.v16n2a9

Annals of

Pure and Applied

Mathematics

\title{
Profit Maximization Solid Transportation Problem with Gaussian Type-2 Fuzzy Environments Sharmistha Jana ${ }^{1}$, Barun Das ${ }^{2}$, Goutam Panigrahi ${ }^{3}$ and Manoranjan Maiti ${ }^{4}$ \\ ${ }^{1}$ Department of Mathematics, Midnapore College (Autonomous) Midnapore \\ West Bengal-721101, India, email: sharmistha792010@gmail.com \\ ${ }^{2}$ Department of Mathematics, Sidho Kanho Birsha University \\ Purulia, West Bengal-723104, India email: bdasskbu@gmail.com \\ ${ }^{3}$ Department of Mathematics, National Institute of Technology Durgapur \\ West Bengal-713209, India email: panigrahi_goutam@ rediffmail.com \\ ${ }^{4}$ Department of Applied Mathematics with Oceanology and Computer Programming, \\ Vidyasagar University, Midnapore-721102, India \\ email: mmaiti2005@yahoo.co.in \\ ${ }^{1}$ Corresponding author
}

Received 18 December 2017; accepted 29 January 2018

\begin{abstract}
Abstruct. A new concept to solve profit maximization transportation problem has been proposed while shipping some goods from a source to destination. In this paper, two transportation schemes have been designed. We consider maximization of the total profit in these two models. In model-I, all variables are considered as Gaussion Type-2 Fuzzy variable, while model -II considered all costs and prices (i.e. purchase cost, selling price, transportation cost and procurement cost) are Gaussion Type-2 Fuzzy variable. The Type-2 fuzzy parameters are transform into Type-I fuzzy parameters with the help of CV-based reduction method. Genetic Algorithm (GA) has been applied to solve the proposed models. Finally, an illustration is presented numerically to demonstrate the experimental results.
\end{abstract}

Keyword: Transportation problem, Critical value, Gaussion Type-2 Fuzzy variables, Genetic algorithm, Reduction method.

AMS Mathematics Subject Classification (2010): 90B05

1. Introduction

In today's rapid changing and competitive business the enterprize are facing a continuous pressure to find better ways and minimum cost to deliver goods to customers. Deliver the products with profit-maximization and time minimization manner become more challenging. Designing a powerful transportation model which provide an effective solution is a challenging job. In reality, transportation problems are developed as a multiobjective problems $[13,20]$. Several objectives may be considered as: delivery time, transportation in good condition, user availability, product impairment, and so on.

Transportation problems(TP) is one of the optimization problems most widely used by private and public sectors. The transportation model was introduced developed 
Sharmistha Jana, Barun Das, Goutam Panigrahi and Manoranjan Maiti

by Hithcock [10] and explain in details by Koopmans [11].

Appa [1] discussed about the different variations in transportation problems. Ramakrishna [14] has describe a variation of vogel's approximation method for finding an initial feasible solution to the transportation problem. Arsham and Khan [2] have developed an algorithm which is faster than simplex. Gass [7] has described different types of TP problem. Adlakham et al.[8,9] and Palekar et al. [12] reviewed briefly the fixed charge transportation problem followed by Barr et al. [3]. Patel and tripathy [12] discussed multi-index TP,where as in 1955, Shell[13] first introduced the concept of multi-dimensional TP(i.e STP). Shell [16] first expressed the solid transportation problem (STP) and Haley [17] formulated a solution for STP. Bit et al. [4] proposed an effective solution with fuzzy to solve multi-objective STP. Some Travelling salesman problems (TSP) has been solved through Fuzzy Inference Method by [19] and Hybrid Algorithm by [18].

Type-2 fuzzy sets are used due to its flexibility and degrees of freedom and it is treated as three dimension. so, type- 2 sets are more efficient for modelling uncertain problem accurately than type- 1 fuzzy variable. The logical operations of type-2 fuzzy were developed by Mizumoto and Tanaka [15] and Dubois and Prade [7]. Later on, good number of research works were developed on the property of type- 2 fuzzy variables $[5,6,7]$ and its application have been presented $[4,8,9]$.

The present paper mainly investigates the following things:

- A computationally efficient defuzzification process of type-2 fuzzy parameters is presented.

- Though TPs with type-1 fuzzy parameters are discussed by many researchers, transportation problems of type-2 fuzzy variable are designed and solved. formulated.

- Chance-constrained programming model with type-2 fuzzy variables is

Here, we have presented profit maximization STP with Gaussian type-2 fuzzy variables. Transportation costs, demands at destination, conveyance procurement cost and capacities all are considered Gaussian type-2 fuzzy variables.

\section{Notations and assumptions of proposed models}

The assumptions and notations are introduced to construct the models are given below:

\subsection{Notations}

- $A_{i}$ : amount of available product at $\mathrm{i}$-th origin.

- $B_{j}$ : demand of products at the $\mathrm{j}$-th end point.

- $C_{i j k}$ : unit cost of transportation to carry product from i-th origin to j-th

destination through k-th conveyance.

- $S_{j}$ : price of selling at the $\mathrm{j}$-th end point.

- $P_{i}$ : purchasing cost at the i-th source.

- $x_{i j k}$ : the amount of product transported from $\mathrm{i}$-th source to $\mathrm{j}$-th destination through $\mathrm{k}$-th conveyance.

- $B M_{i}$ : unit procurement cost for product from $\mathrm{i}$-th source to $\mathrm{j}$-th destination by 
Profit Maximization Solid Transportation Problem with Gaussian Type-2 Fuzzy Environments

k-th conveyed.

- $E_{k}$ : The product amount which conveyed by k-th conveyance.

- $f_{i j k}$ : fixed charged transportation cost to transport unit product from i-th source to $\mathrm{j}$-th destination by $\mathrm{k}$-th conveyance.

- $R$ : Number of items.

- $M$ : Sources numbers or origins.

- $N$ : Destinations numbers.

- $K$ : Conveyances numbers.

\subsection{Assumptions}

- Problems are unbalanced in these models.

- Considering quantity of products are transported from $\mathrm{i}$-th origin to $\mathrm{j}$-th destination through various ways of k-th conveyances is $x_{i j k}>0$ then we define $y_{i j k}$ as shown below :

$$
y_{i j k}= \begin{cases}1 & 2.2 \mathrm{~cm} \text { for } x_{i j k}>0 \\ 0 & 2.2 \mathrm{~cm} \text { otherwise }\end{cases}
$$

\subsection{Model formulation}

In balanced STP, the total amounts of suppliers, demands and conveyance are equal in real situation. But the balanced conditions does not hold. Here consider that enough items is in the origin to meet the demand of each and every end point conveyances have the capacity to carry item to meet of the demand at each end point. In this model profit maximization STP of unit transportation costs, availabilities, demands, conveyance capability,cost of purchasing,price of selling and procurement cost has been considered in our approach.

\subsection{Model-1}

Price of selling, cost of purchase, unit transportation cost, unit procurement cost, origin, demands and Conveyance capability are considered as Gaussian fuzzy type-2 variables

$$
\begin{aligned}
& \operatorname{Maxf}_{1}=\sum_{i=1}^{M} \sum_{j=1}^{N} \sum_{k=1}^{K}\left\{\tilde{S}_{j}-\tilde{P}_{i}-\tilde{C}_{i j k}-\tilde{B} M_{i}\right\} x_{i j k} \\
& \text { subject to } \sum_{j=1}^{N} \sum_{k=1}^{K} x_{i j k} \leq \tilde{A}_{i} \quad i=1,2, \ldots M, \\
& \sum_{i=1}^{M} \sum_{k=1}^{K} x_{i j k} \geq \tilde{B}_{j} \quad j=1,2, \ldots N, \\
& \sum_{i=1}^{M} \sum_{j=1}^{N} x_{i j k} \leq \tilde{E}_{k} \\
& x_{i j k} \geq 0
\end{aligned}
$$


Sharmistha Jana, Barun Das, Goutam Panigrahi and Manoranjan Maiti

$$
\text { where } w\left(x_{i j k}\right)=\left\{\begin{array}{l}
1, \text { if } x_{i j k}>0 ; \\
0, \text { otherwise } \forall i, j, k
\end{array}\right.
$$

\subsection{Defuzzification of Gaussian type-2 fuzzy variables}

$$
\begin{array}{lc}
\operatorname{Max} \bar{f} & \operatorname{Cr}\left\{\sum_{i=1}^{M} \sum_{j=1}^{N} \sum_{k=1}^{K}\left\{\tilde{S}_{j}-\tilde{P}_{i}-\tilde{C}_{i j k}-\tilde{B} M_{i}\right\} x_{i j k} \geq \bar{f}\right\} \geq \alpha \\
\operatorname{Cr}\left\{\sum_{j=1}^{N} \sum_{k=1}^{K} x_{i j k} \leq \tilde{A}_{i}\right\} \geq \alpha_{i} & i=1,2, \ldots M, \\
\operatorname{Cr}\left\{\sum_{j=1}^{N} \sum_{k=1}^{K} x_{i j k} \geq \tilde{B}_{j}\right\} \geq \beta_{j} & j=1,2, \ldots N, \\
\operatorname{Cr}\left\{\sum_{i=1}^{M} \sum_{j=1}^{N} x_{i j k} \leq \tilde{E}_{k}\right\} \geq \gamma_{k} & k=1,2, \ldots K, x_{i j k} \geq 0, \forall i, j, k .
\end{array}
$$

Here $\operatorname{Maxf}_{1}$ indicate the maximum value and the objective function accomplisn with generalized credibility $\alpha(0<\alpha \leq 1) . \alpha_{i}, \beta_{j}, \gamma_{k}\left(0<\alpha_{i}, \beta_{j}, \gamma_{k} \leq 1\right)$ which are the present generalized credibility satisfaction level of the origin and end point restriction respectively for all $\mathrm{i}, \mathrm{j}, \mathrm{k}$.

\section{Case i:}

When $\alpha \varepsilon[0,0.25]$, then the parametric problem of the model representation (5) as:

$$
\begin{aligned}
& \operatorname{Max} \bar{f} \\
& \text { s.t } \quad \sum_{i=1}^{M} \sum_{j=1}^{N} \sum_{k=1}^{K}\left(\left(\mu_{\tilde{s}_{j}}-\sigma_{\tilde{s}_{j}} \sqrt{\left.2 \ln \left(1+(1-4 \alpha) \theta_{r, \tilde{s}_{j}}\right)-2 \ln 2 \alpha\right)} x_{i j k}\right.\right. \\
& -\left(\mu_{\tilde{p}_{i}}-\sigma_{\tilde{p}_{i}} \sqrt{\left.2 \ln \left(1+(1-4 \alpha) \theta_{r, \tilde{p}_{i}}\right)-2 \ln 2 \alpha\right)} x_{i j k}\right. \\
& -\left(\mu_{\tilde{c}_{i j k}}-\sigma_{\tilde{c}_{i j k}} \sqrt{\left.2 \ln \left(1+(1-4 \alpha) \theta_{r, \tilde{c}_{i j k}}\right)-2 \ln 2 \alpha\right)} x_{i j k}\right. \\
& -\left(\mu_{\tilde{B} M_{i}}-\sigma_{\tilde{B} M_{i}} \sqrt{\left.2 \ln \left(1+(1-4 \alpha) \theta_{r, \tilde{B} M_{i}}\right)-2 \ln 2 \alpha\right)} x_{i j k}\right) \geq \bar{f} \\
& \text { and } \quad \sum_{j=1}^{N} \sum_{k=1}^{K} x_{i j k} \leq\left(\mu_{\tilde{a}_{i}}-\sigma_{\tilde{a}_{i}} \sqrt{\left.2 \ln \left(1+\left(1-4 \alpha_{i}\right) \theta_{r, \tilde{a}_{i}}\right)-2 \ln 2 \alpha_{i}\right)}\right. \text {, } \\
& i=1,2,3, \ldots . M \\
& \sum_{i=1}^{M} \sum_{k=1}^{K} x_{i j k} \geq\left(\mu_{\tilde{b}_{j}}-\sigma_{\tilde{b}_{j}} \sqrt{\left.2 \ln \left(1+\left(1-4 \beta_{j}\right) \theta_{r, \tilde{b}_{j}}\right)-2 \ln 2 \beta_{j}\right)},\right. \\
& j=1,2,3, \ldots . N \\
& \sum_{i=1}^{M} \sum_{j=1}^{N} x_{i j k} \leq\left(\mu_{\tilde{e}_{k}}-\sigma_{\tilde{e}_{k}} \sqrt{\left.2 \ln \left(1+\left(1-4 \gamma_{k}\right) \theta_{r, \tilde{e}_{k}}\right)-2 \ln 2 \gamma_{k}\right)}\right. \text {, } \\
& k=1,2,3, \ldots . K
\end{aligned}
$$


Profit Maximization Solid Transportation Problem with Gaussian Type-2 Fuzzy Environments

Case ii:

When $\alpha \varepsilon[2.5,0.5]$, then the parametric problem of the model representation (5) as:

$\operatorname{Max} \bar{f}$

$$
\begin{aligned}
& \text { s.t } \quad \sum_{i=1}^{M} \sum_{j=1}^{N} \sum_{k=1}^{K}\left(\left(\mu_{\tilde{s}_{j}}-\sigma_{\tilde{s}_{j}} \sqrt{\left.2 \ln \left(1+(4 \alpha-1) \theta_{r, \tilde{s}_{j}}\right)-2 \ln \left(2 \alpha+(4 \alpha-1) \theta_{1, s_{j}}\right)\right)} x_{i j k}\right.\right. \\
& -\left(\mu_{\tilde{p}_{i}}-\sigma_{\tilde{p}_{i}} \sqrt{\left.2 \ln \left(1+(4 \alpha-1) \theta_{r, \tilde{p}_{i}}\right)-2 \ln \left(2 \alpha+(4 \alpha-1) \theta_{1, p_{i}}\right)\right)} x_{i j k}\right. \\
& -\left(\mu_{\tilde{c}_{i j k}}-\sigma_{\tilde{c}_{i j k}} \sqrt{\left.2 \ln \left(1+(4 \alpha-1) \theta_{r, \tilde{c}_{i j k}}\right)-2 \ln \left(2 \alpha+(4 \alpha-1) \theta_{1, c_{i j k}}\right)\right)} x_{i j k}\right. \\
& -\left(\mu_{\tilde{B} M_{i}}-\sigma_{\tilde{B} M_{i}} \sqrt{\left.\left.2 \ln \left(1+(4 \alpha-1) \theta_{r, \tilde{B} M_{i}}\right)-2 \ln (2 \alpha+4 \alpha-1) \theta_{1, B M_{i}}\right)\right)} x_{i j k}\right) \geq \bar{f} \\
& \text { and } \sum_{j=1}^{N} \sum_{k=1}^{K} x_{i j k} \leq\left(\mu_{\tilde{a}_{i}}-\sigma_{\tilde{a}_{i}} \sqrt{\left.2 \ln \left(1+\left(4 \alpha_{i}-1\right) \theta_{r, \tilde{a}_{i}}\right)-2 \ln \left(2 \alpha_{i}+\left(4 \alpha_{i}-1\right) \theta_{1, a_{i}}\right)\right)}\right. \text {, } \\
& i=1,2,3, \ldots . M \\
& \sum_{i=1}^{M} \sum_{k=1}^{K} x_{i j k} \geq\left(\mu_{\tilde{b}_{j}}-\sigma_{\tilde{b}_{j}} \sqrt{\left.2 \ln \left(1+\left(4 \beta_{j}-1\right) \theta_{r, \tilde{b}_{j}}\right)-2 \ln \left(2 \beta_{j}+\left(4 \beta_{j}-1\right) \theta_{r, b_{j}}\right)\right)},\right. \\
& j=1,2,3, \ldots . . N \\
& \sum_{i=1}^{M} \sum_{j=1}^{N} x_{i j k} \leq\left(\mu_{\tilde{e}_{k}}-\sigma_{\tilde{e}_{k}} \sqrt{\left.2 \ln \left(1+\left(4 \gamma_{k}-1\right) \theta_{r, \tilde{e}_{k}}\right)-2 \ln \left(2 \gamma_{k}+\left(4 \gamma_{k}-1\right) \theta_{1, e_{k}}\right)\right)},\right. \\
& k=1,2,3, \ldots . K
\end{aligned}
$$

Case iii:

When $\alpha \varepsilon[0.5,7.5]$, then the parametric problem of the model representation (5) as:

$\operatorname{Max} \bar{f}$

s.t $\quad \sum_{i=1}^{M} \sum_{j=1}^{N} \sum_{k=1}^{K}\left(\left(\mu_{\tilde{s}_{j}}+\sigma_{\tilde{s}_{j}} \sqrt{\left.2 \ln \left(1+(3-4 \alpha) \theta_{r, \tilde{s}_{j}}\right)-2 \ln \left(2(1-\alpha)+(3-4 \alpha) \theta_{1, \tilde{s}_{j}}\right)\right)} x_{i j k}\right.\right.$

$-\left(\mu_{\tilde{p}_{i}}+\sigma_{\tilde{p}_{i}} \sqrt{\left.2 \ln \left(1+(3-4 \alpha) \theta_{r, \tilde{p}_{i}}\right)-2 \ln \left(2(1-\alpha)+(3-4 \alpha) \theta_{1, \tilde{p}_{i}}\right)\right)} x_{i j k}\right.$

$-\left(\mu_{\tilde{c}_{i j k}}+\sigma_{\tilde{c}_{i j k}} \sqrt{\left.2 \ln \left(1+(3-4 \alpha) \theta_{r, \tilde{c}_{i j k}}\right)-2 \ln \left(2(1-\alpha)+(3-4 \alpha) \theta_{1, \tilde{c}_{i j k}}\right)\right)} x_{i j k}\right.$

$-\left(\mu_{\tilde{B} M_{i}}+\sigma_{\tilde{B} M_{i}} \sqrt{\left.2 \ln \left(1+(3-4 \alpha) \theta_{r, \tilde{B} M_{i}}\right)-2 \ln \left(2(1-\alpha)+(3-4 \alpha) \theta_{1, \tilde{B} M_{i}}\right)\right)} x_{i j k}\right) \geq \bar{f}$

and $\sum_{j=1}^{N} \sum_{k=1}^{K} x_{i j k} \leq\left(\mu_{\tilde{a}_{i}}+\sigma_{\tilde{a}_{i}} \sqrt{\left.2 \ln \left(1+\left(3-4 \alpha_{i}\right) \theta_{r, \tilde{s}_{j}}\right)-2 \ln \left(2\left(1-\alpha_{i}\right)+\left(3-4 \alpha_{i}\right) \theta_{1, \tilde{s}_{j}}\right)\right)}\right.$,

$i=1,2,3, \ldots . M$

$\sum_{i=1}^{M} \sum_{k=1}^{K} x_{i j k} \geq\left(\mu_{\tilde{b}_{j}}+\sigma_{\tilde{b}_{j}} \sqrt{\left.2 \ln \left(1+\left(3-4 \beta_{j}\right) \theta_{r, \tilde{s}_{j}}\right)-2 \ln \left(2\left(1-\beta_{j}\right)+(3-4 \alpha) \theta_{1, \tilde{s}_{j}}\right)\right)}\right.$,

$j=1,2,3, \ldots . N$ 
Sharmistha Jana, Barun Das, Goutam Panigrahi and Manoranjan Maiti

$\sum_{i=1}^{M} \sum_{j=1}^{N} x_{i j k} \leq\left(\mu_{\tilde{e}_{k}}+\sigma_{\tilde{e}_{k}} \sqrt{\left.2 \ln \left(1+\left(3-4 \gamma_{k}\right) \theta_{r, \tilde{s}_{j}}\right)-2 \ln \left(2\left(1-\gamma_{k}\right)+\left(3-4 \gamma_{k}\right) \theta_{1, \tilde{s}_{j}}\right)\right)}\right.$,

$k=1,2,3, \ldots . . K$

Case iv:

When $\alpha \varepsilon[0.75,1]$, then the parametric problem of the model representation (5) as:

$\operatorname{Max} \bar{f}$

$$
\begin{aligned}
& \text { s.t } \quad \sum_{i=1}^{M} \sum_{j=1}^{N} \sum_{k=1}^{K}\left(\left(\mu_{\tilde{s}_{j}}+\sigma_{\tilde{s}_{j}} \sqrt{2 \ln \left(1+(4 \alpha-3) \theta_{r, \tilde{s}_{j}}\right)-2 \ln (2(\alpha-1))} x_{i j k}\right.\right. \\
& -\left(\mu_{\tilde{p}_{i}}+\sigma_{\tilde{p}_{i}} \sqrt{2 \ln \left(1+(4 \alpha-3) \theta_{r, \tilde{p}_{i}}\right)-2 \ln (2(1-\alpha))} x_{i j k}\right. \\
& -\left(\mu_{\tilde{c}_{i j k}}+\sigma_{\tilde{c}_{i j k}} \sqrt{2 \ln \left(1+(4 \alpha-3) \theta_{r, \tilde{c}_{j j k}}\right)-2 \ln (2(1-\alpha))} x_{i j k}\right. \\
& -\left(\mu_{\tilde{B} M_{i}}+\sigma_{\tilde{B} M_{i}} \sqrt{2 \ln \left(1+(4 \alpha-3) \theta_{r, \tilde{B} M_{i}}\right)-2 \ln (2(1-\alpha))} x_{i j k}\right) \geq \bar{f}
\end{aligned}
$$

and $\sum_{j=1}^{N} \sum_{k=1}^{K} x_{i j k} \leq\left(\mu_{\tilde{a}_{i}}+\sigma_{\tilde{a}_{i}} \sqrt{2 \ln \left(1+\left(4 \alpha_{i}-3\right) \theta_{r, \tilde{a}_{i}}\right)-2 \ln \left(2\left(\alpha_{i}-1\right)\right)}\right.$,

$i=1,2,3, \ldots . M$

$\sum_{i=1}^{M} \sum_{k=1}^{K} x_{i j k} \geq\left(\mu_{\tilde{b}_{j}}+\sigma_{\tilde{b}_{j}} \sqrt{2 \ln \left(1+\left(4 \beta_{j}-3\right) \theta_{r, \tilde{b}_{j}}\right)-2 \ln \left(2\left(1-\beta_{j}\right)\right)}\right.$,

$j=1,2,3, \ldots . N$

$\sum_{i=1}^{M} \sum_{j=1}^{N} x_{i j k} \leq\left(\mu_{\tilde{e}_{k}}+\sigma_{\tilde{e}_{k}} \sqrt{2 \ln \left(1+\left(4 \gamma_{k}-3\right) \theta_{r, \tilde{e}_{k}}\right)-2 \ln \left(2\left(\gamma_{k}-1\right)\right)}\right.$,

$k=1,2,3, \ldots . K$

\subsection{Model-2}

Price of selling, cost of purchase, unit transportation cost, unit procurement cost are treated as Gaussian type-2 fuzzy variables and source, demands and conveyance capacities are crisp

$$
\begin{aligned}
& \operatorname{Maxf}_{1}=\sum_{i=1}^{M} \sum_{j=1}^{N} \sum_{k=1}^{K}\left\{\tilde{S}_{j}-\tilde{P}_{i}-\tilde{C}_{i j k}-\tilde{B} M_{i}\right\} x_{i j k} \\
& \text { subject to } \sum_{j=1}^{N} \sum_{k=1}^{K} x_{i j k} \leq A_{i} \quad i=1,2, \ldots M, \\
& \sum_{i=1}^{M} \sum_{k=1}^{K} x_{i j k} \geq B_{j} \quad j=1,2, \ldots N, \\
& \sum_{i=1}^{M} \sum_{j=1}^{N} x_{i j k} \leq E_{k} \quad k=1,2, \ldots K,
\end{aligned}
$$


Profit Maximization Solid Transportation Problem with Gaussian Type-2 Fuzzy Environments

$$
x_{i j k} \geq 0
$$

where $w\left(x_{i j k}\right)=\left\{\begin{array}{l}1 \text { if } x_{i j k}^{r}>0 \text { for at least oner } \\ 0 \text { if } x_{i j k}^{r}=0 \text { for allr }\end{array}\right.$

2.6.1. Case i:

When $\alpha \varepsilon[0,0.25]$, then the parametric problem of the model representation (5) as:

$$
\begin{aligned}
& \operatorname{MaxTP}=\sum_{i=1}^{M} \sum_{j=1}^{N} \sum_{k=1}^{K}\left(\left(\mu_{\tilde{s}_{j}}-\sigma_{\tilde{s}_{j}} \sqrt{\left.2 \ln \left(1+(1-4 \alpha) \theta_{r, \tilde{s}_{j}}\right)-2 \ln 2 \alpha\right)} x_{i j k}\right.\right. \\
& -\left(\mu_{\tilde{p}_{i}}-\sigma_{\tilde{p}_{i}} \sqrt{\left.2 \ln \left(1+(1-4 \alpha) \theta_{r, \tilde{p}_{i}}\right)-2 \ln 2 \alpha\right)} x_{i j k}\right. \\
& -\left(\mu_{\tilde{c}_{i j k}}-\sigma_{\tilde{c}_{i j k}} \sqrt{\left.2 \ln \left(1+(1-4 \alpha) \theta_{r, \tilde{c}_{i j k}}\right)-2 \ln 2 \alpha\right)} x_{i j k}\right. \\
& -\left(\mu_{\tilde{B} M_{i}}-\sigma_{\tilde{B} M_{i}} \sqrt{\left.2 \ln \left(1+(1-4 \alpha) \theta_{r, \tilde{B} M_{i}}\right)-2 \ln 2 \alpha\right)} x_{i j k}\right) \geq \bar{f} \\
& \quad \text { s.t } \quad(11)-(13)
\end{aligned}
$$

2.6.2. Case ii:

When $\alpha \varepsilon[2.5,0.5]$, then the parametric problem of the model representation (5) as:

$$
\begin{aligned}
\operatorname{MaxTP}= & \sum_{i=1}^{M} \sum_{j=1}^{N} \sum_{k=1}^{K}\left(\left(\mu_{\tilde{s}_{j}}-\sigma_{\tilde{s}_{j}} \sqrt{\left.2 \ln \left(1+(4 \alpha-1) \theta_{r, \tilde{s}_{j}}\right)-2 \ln \left(2 \alpha+(4 \alpha-1) \theta_{1, s_{j}}\right)\right)} x_{i j k}\right.\right. \\
& -\left(\mu_{\tilde{p}_{i}}-\sigma_{\tilde{p}_{i}} \sqrt{\left.2 \ln \left(1+(4 \alpha-1) \theta_{r, \tilde{p}_{i}}\right)-2 \ln \left(2 \alpha+(4 \alpha-1) \theta_{1, p_{i}}\right)\right)} x_{i j k}\right. \\
& -\left(\mu_{\tilde{c}_{i j k}}-\sigma_{\tilde{c}_{i j k}} \sqrt{\left.2 \ln \left(1+(4 \alpha-1) \theta_{r, \tilde{c}_{i j k}}\right)-2 \ln \left(2 \alpha+(4 \alpha-1) \theta_{1, c_{i j k}}\right)\right)} x_{i j k}\right. \\
& -\left(\mu_{\tilde{B} M_{i}}-\sigma_{\tilde{B} M_{i}} \sqrt{\left.2 \ln \left(1+(4 \alpha-1) \theta_{r, \tilde{B} M_{i}}\right)-2 \ln \left(2 \alpha+(4 \alpha-1) \theta_{1, B M_{i}}\right)\right)} x_{i j k}\right) \geq \bar{f}
\end{aligned}
$$

s.t $\quad(11)-(13)$

\subsubsection{Case iii:}

When $\alpha \varepsilon[0.5,7.5]$, then the parametric problem of the model representation (5) as:

$$
\begin{aligned}
& \operatorname{MaxTP}=\sum_{i=1}^{M} \sum_{j=1}^{N} \sum_{k=1}^{K}\left(\left(\mu_{\tilde{s}_{j}}+\sigma_{\tilde{s}_{j}} \sqrt{\left.2 \ln \left(1+(3-4 \alpha) \theta_{r, \tilde{s}_{j}}\right)-2 \ln \left(2(1-\alpha)+(3-4 \alpha) \theta_{1, \tilde{s}_{j}}\right)\right)} x_{i j k}\right.\right. \\
& -\left(\mu_{\tilde{p}_{i}}+\sigma_{\tilde{p}_{i}} \sqrt{\left.2 \ln \left(1+(3-4 \alpha) \theta_{r, \tilde{p}_{i}}\right)-2 \ln \left(2(1-\alpha)+(3-4 \alpha) \theta_{1, \tilde{p}_{i}}\right)\right)} x_{i j k}\right. \\
& -\left(\mu_{\tilde{c}_{i j k}}+\sigma_{\tilde{c}_{i j k}} \sqrt{\left.2 \ln \left(1+(3-4 \alpha) \theta_{r, \tilde{c}_{i j k}}\right)-2 \ln \left(2(1-\alpha)+(3-4 \alpha) \theta_{1, \tilde{c}_{i j k}}\right)\right)} x_{i j k}\right. \\
& -\left(\mu_{\tilde{B} M_{i}}+\sigma_{\tilde{B}_{i}} \sqrt{\left.2 \ln \left(1+(3-4 \alpha) \theta_{r, \tilde{B} M_{i}}\right)-2 \ln \left(2(1-\alpha)+(3-4 \alpha) \theta_{1, \tilde{B} M_{i}}\right)\right)} x_{i j k}\right) \geq \bar{f} \\
& \quad s . t
\end{aligned}
$$

\subsubsection{Case iv:}


Sharmistha Jana, Barun Das, Goutam Panigrahi and Manoranjan Maiti

When $\alpha \varepsilon[0.75,1]$, then the parametric problem of the model representation (5) as:

$$
\begin{aligned}
\operatorname{MaxTP} & =\sum_{i=1}^{M} \sum_{j=1}^{N} \sum_{k=1}^{K}\left(\left(\mu_{\tilde{s}_{j}}+\sigma_{\tilde{s}_{j}} \sqrt{2 \ln \left(1+(4 \alpha-3) \theta_{r, \tilde{s}_{j}}\right)-2 \ln (2(\alpha-1))} x_{i j k}\right.\right. \\
& -\left(\mu_{\tilde{p}_{i}}+\sigma_{\tilde{p}_{i}} \sqrt{2 \ln \left(1+(4 \alpha-3) \theta_{r, \tilde{p}_{i}}\right)-2 \ln (2(1-\alpha))} x_{i j k}\right. \\
& -\left(\mu_{\tilde{c}_{i j k}}+\sigma_{\tilde{c}_{i j k}} \sqrt{2 \ln \left(1+(4 \alpha-3) \theta_{r, \tilde{c}_{i j k}}\right)-2 \ln (2(1-\alpha))} x_{i j k}\right. \\
& -\left(\mu_{\tilde{B} M_{i}}+\sigma_{\tilde{B} M_{i}} \sqrt{2 \ln \left(1+(4 \alpha-3) \theta_{r, \tilde{B} M_{i}}\right)-2 \ln (2(1-\alpha))} x_{i j k}\right) \geq \bar{f} \\
\text { s.t } \quad & (11)-(13)
\end{aligned}
$$

\section{Solution procedures}

Genetic Algorithm (GA) has been used to solve the problem of given model. GA is used to find optimization through heuristic search process that corresponds natural selection. Here population is as a set of feasible solutions of proposed problem. Genotype is called as considered member of population, a chromosome, a string or a permutation. A GA performed three different operations - reproduction, crossover and mutation.

\subsection{Parameters}

The different parameters are considered to solve the problem through GA as follows.

$(M A X G E N)$-number of generation (set 5000)

(POPSIZE) -size of population (set 100)

(PXOVER) - probability of crossover (set 0.6)

$(P M U)$-probability of mutation (set 0.2 ).

\subsection{Representation of chromosome}

The variables in this proposed models are non-linear. So, a real - number is used to represent the chromosome to solve the proposed model. Many non-linear real problems used binary vectors but those were not effective.

\subsection{Reproduction}

To evaluate the chromosome, parents are randomly selected. The boundaries dependent variables, independent variables are determined from all (here 16) variables to initialize the population.

\subsection{Crossover}

The main operator of GA is Crossover.It is used to exchange the parent's characteristics and communicate to the children. It may happen in two steps:

- Selection for crossover: A random number $r$ is generated for each solution of $P^{1}(T)$ from the range [0..1]. The solution is considered for crossover, if $\mathrm{r}<\mathrm{p}_{c}$ where $\mathrm{p}_{c}$ is crossover probability .

- Crossover process: After selection some solution, Crossover has been applied . The random number $\mathrm{c}$ has been taken from the range [0..1] for the pair of solutions $\mathrm{Y}_{1}$, 
Profit Maximization Solid Transportation Problem with Gaussian Type-2 Fuzzy Environments

$\mathrm{Y}_{2} . \mathrm{Y}_{11}$ and $\mathrm{Y}_{21}$ are calculated using $\mathrm{Y}_{1}, \mathrm{Y}_{2}$ as follows :

where $\mathrm{Y}_{11}=\mathrm{c} \mathrm{Y}_{1}+(1-\mathrm{c}) \mathrm{Y}_{2}, \mathrm{Y}_{21}=\mathrm{cY} \mathrm{Y}_{2}+(1-\mathrm{c}) \mathrm{Y}_{1}$, where $Y_{11}, Y_{21}$ must meet the

problem constraints.

\subsection{Mutation}

To recover any loss of some important characteristics, we need to perform mutation operation .It also used for maintain population diversity .It is done in two steps:

- Mutation Selection: A random number $r$ is generated for each solution of $P^{1}(T)$ from the range [0..1]. The solution is considered for mutation, If $r<p_{m}$. Where $p_{m}$ is the mutation probability .

- Mutation process: A random number $\mathrm{r}$ is selected with in the range [1..K].

Then by replacement of $\mathrm{x}_{r}$ within $\mathrm{r}^{\text {th }}$ component of $\mathrm{X}$ they are random number. We get a solution $X=\left(x_{1}, x_{2}, \ldots . x_{k}\right)$, which is a solution through mutation.

\subsection{Evaluation}

The evaluation function is used to solve this problem is eval $\left(V_{i}\right)=$ objective function value

Through Roulette wheel selection chromosome .Here better chromosome has been selected from the population to generate the new chromosomes. Now new improved better chromosomes are generated through arithmetic crossover and mutation. The steps of the proposed algorithm is given below:

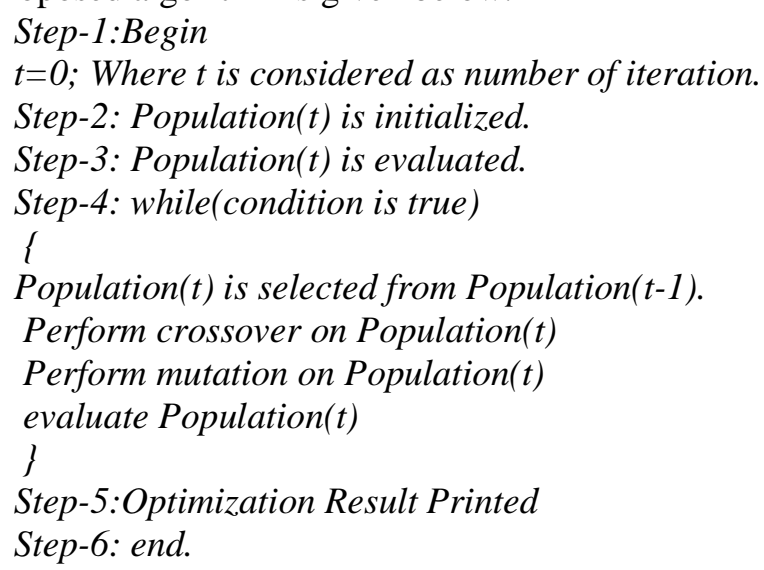

\section{Numerical experiments}

To present the relevancy and utility of the proposed model, a numerical illustration with three sources and three destination and three convenances are considered in this models. The model described above is coded in GA to solve the profit maximization solid transportation problem. 
Sharmistha Jana, Barun Das, Goutam Panigrahi and Manoranjan Maiti

Table 1: Gaussian T2 fuzzy unit transportation costs

\begin{tabular}{|l|l|l|l|}
\hline 1 & $(3,1.0 ; 0.7,1.0)$ & $(4,1.5 ; 0.5,1.0)$ & $(2,0.8 ; 0.7,1.0)$ \\
\hline 2 & $(5,1.5 ; 0.5,1.5)$ & $(4,1.0 ; 1.4,2.0)$ & $(3,1.2 ; 0.5,0.9)$ \\
\hline 3 & $(3,1.2 ; 0.8,1.0)$ & $(5,1.9 ; 0.5,1.0)$ & $(6,1.0 ; 1.4,2.0)$ \\
\hline 1 & $(6,2.0 ; 0.7,1.0)$ & $(4,1.5 ; 0.5,1.5)$ & $(6,2.0 ; 0.7,1.0)$ \\
\hline 2 & $(5,2.5 ; 0.5,1.5)$ & $(6,1.3 ; 1.4,2.2)$ & $(8,1.2 ; 0.5,0.9)$ \\
\hline 3 & $(3,0.8 ; 0.6,1.0)$ & $(9,1.9 ; 1.0,1.4)$ & $(7,1.0 ; 1.0,1.0)$ \\
\hline 1 & $(3,1.0 ; 0.7,1.0)$ & $(4,1.5 ; 0.5,1.0)$ & $(2,0.8 ; 0.7,1.0)$ \\
\hline 2 & $(5,1.5 ; 0.5,1.5)$ & $(4,1.0 ; 1.4,2.0)$ & $(3,1.2 ; 0.5,0.9)$ \\
\hline 3 & $(3,1.2 ; 0.8,1.0)$ & $(5,1.9 ; 0.5,1.0)$ & $(6,1.0 ; 1.0,1.0)$ \\
\hline
\end{tabular}

Table 2: Solid transportation problem parameters

\begin{tabular}{|c|c|c|c|c|c|}
\hline $\mathrm{i}$ & Source & $\mathrm{j}$ & Demand & $\mathrm{k}$ & $\begin{array}{c}\text { Capacity of } \\
\text { Conveyance }\end{array}$ \\
\hline 1 & $(30,1.5 ; 0.8,1.0)$ & 1 & $(23,2.1 ; 0.5,0.8)$ & 1 & $(43,2.1 ; 1.2,1.6)$ \\
\hline 2 & $(31,1.2 ; 0.1,1.0)$ & 2 & $(21,1.1 ; 0.5,0.8)$ & 2 & $(35,2.1 ; 1.0,1.6)$ \\
\hline 3 & $(27,1.8 ; 0.8,1.0)$ & 3 & $(20,2.1 ; 0.5,0.8)$ & 3 & $(30,1.5 ; 0.8,1.6)$ \\
\hline
\end{tabular}

Table 3: Selling Prices and Purchase costs

\begin{tabular}{|c|c|}
\hline Selling Price & Purchase costs \\
\hline$(45,1.0 ; 0.8,1.8)$ & $(7,1.2 ; 0.9,1.5)$ \\
\hline$(58,1.0 ; 0.8,1.8)$ & $(9,1.2 ; 0.9,1.5)$ \\
\hline$(50,1.0 ; 0.8,1.8)$ & $(8,1.2 ; 0.9,1.5)$ \\
\hline
\end{tabular}

Table 4: Procurement cost per product

\begin{tabular}{|c|c|c|c|}
\hline Product & $\mathrm{P}(1)$ & $\mathrm{P}(2)$ & $\mathrm{P}(3)$ \\
\hline & $(4.5,1.0 ; 0.8,1.8)$ & $(3.1,1.2 ; 0.9,1.5)$ & $(2.23,1.0 ; 1.1,1.5)$ \\
\hline
\end{tabular}

\section{Discussion}

We obtained determined compromise solutions from the experiment which are distinct with different degrees. The performance of this model has been shown through the experimental result in Table- 5. The obtained results demonstrate the applicability and managerial insight of the proposed scheme. The proposed algorithm is very effective for searching better solution and we achieve Pareto optimal solutions for managerial decision. Here, we observed that profit of Model-1 is greater than the profit in Model2.GA has been used to shown the crossover of results.It is possible to get the result through the variations of population size, iteration, crossover and mutation.

\section{Practical implication}

A food supplier company of West Bengal, India supply one type of food namely rice from three source points namely Hoogly, Midnapore, and Malda of West Bengal, India by difference conveyances (lorry, truck, train etc) to three destinations Kolkata, Durgapur and Kharagpur. The main aims of the proposed models are to maximize the profit against the market price at different market. Due to fluctuation of fuel price, road tax for different route, political issues, different type of procurement cost in each route 


\section{Profit Maximization Solid Transportation Problem with Gaussian Type-2 Fuzzy Environments}

is not fixed. The transportation cost of carrying one unit $(100 \mathrm{~kg})$ of rice from source to a destination by any convenances is treated as a multi objective multi choice transportation problem. Here, we have considered the parameters of transportation problem through Gaussian type-2 fuzzy environment.

Table 5: Different models results (optimum)

\begin{tabular}{|c|c|c|c|c|}
\hline$\alpha$ & Model & Amount & Profit & Optimum transported amount \\
\hline \multirow{2}{*}{.95} & 1 & 112.189 & 1713.337 & $\begin{array}{c}14.2859,21.7021,5.6732,12.1224,16.2321,6.3129 \\
1.4229,16.6747,21.594 \text { and remaining variables are zero. }\end{array}$ \\
\hline & 2 & 106 & 1592.660 & $\begin{array}{l}\text { 17.6341, 14.3612, } 10.1542,8.4721,2.1212,4.5421,2.3399, \\
13.1232,10.3151,23.3761 \text { and remaining variables are zero. }\end{array}$ \\
\hline \multirow{2}{*}{0.90} & 1 & 115.189 & 1719.418 & $\begin{array}{l}11.5239,23.3124,1.4143,5.432,0.6213,6.5429,16.6352 \\
14.4329,6.5347,26.3159 \text { and remaining variables are zero. }\end{array}$ \\
\hline & 2 & 106 & 1599.658 & $\begin{array}{c}6.3154,34.4325,0.5321,2.7343,21.5665,4.6153 \\
12.1654,1.0623,2.423 \text { and remaining variables are zero. }\end{array}$ \\
\hline \multirow{2}{*}{0.85} & 1 & 115.189 & 1723.972 & $\begin{array}{c}22.7587,42.1129,0.5637,4.7653,13.9659,14.3127,0.9687, \\
14.2129,1.5173,0.4123,26.5321 \text { and remaining variables are zero. }\end{array}$ \\
\hline & 2 & 106 & 1704.880 & $\begin{array}{c}12.1065,13.3219,2.6126,3.5305,2.1237,17.3412,2.5243 \\
12.3452,11.3754,1.5965,2.5422 \text { and remaining variables are zero. }\end{array}$ \\
\hline \multirow{2}{*}{0.80} & 1 & 115.189 & 1732.672 & $\begin{array}{c}21.08734,13.4331,29.5123,2.6541,3.6512,3.6123,10.59 \\
4.5812,2.4523,14.2913,6.1237,12.4123,26.4359,12.341 \\
\text { and remaining variables are zero. }\end{array}$ \\
\hline & 2 & 106 & 1612.186 & $\begin{array}{c}12.0321,39.3421,0.7341,0.9812, .8916,27.3245,2.1284, \\
12.4132,9,0.7831,2.8932,1.6123 \text { and remaining variables are zero. }\end{array}$ \\
\hline
\end{tabular}

7. Comparison with earlier work

It has been observed that few development has been done using STP with profit maximization. Most work has been developed by considering cost minimization. Here we investigate the problem in the angle of profit maximization. This two approach are opposite angle to it is hard to comparison between them. This two is the new development using profit maximization solid transportation problem with Gaussion Type-2 Fuzzy Environments. So, this is a new innovative investigation towards the field of transportation as per our knowledge.

\section{Conclusions and future scope}

A new profit maximization STP with all parameters are considered as Gaussion Type-2 Fuzzy Environments.Here the parameters are supply, demand, capacity of conveyance,price of selling, cost for purchase, cost of transportation and unit Procurement cost. The GA has been used to solve the proposed model and achieve good results.The main contribution are mentation below:

- This is the first attempt in STP with profit maximization.

- Gaussion Type-2 has been used to get accurate result which is more precise than type-1. 
Sharmistha Jana, Barun Das, Goutam Panigrahi and Manoranjan Maiti

- A new concept has been developed using these model. One can apply using time minimization budget constraint, damage item, discount of price, festival offer and so on.

- This model can be extended and formulated with rough, fuzzy rough, intuitionists fuzzy environment etc.

\section{REFERENCES}

1. G.M.Appa, Transportation problem and its varients, Operational Research Quarterly, 24(1) (1973) 79 -99.

2. H.Arsham and A.B.Khan, A simplex algorithm for general transpotation problem:an alternative to stepping stone, Journal of Operational Research Society, 40 (1989) 581-590.

3. R.S.Barr,F.Glover and D.Klingman, A new optimization method for large scal fixed charge transpotation problem, Operations Research, 23 (1981) 448-463.

4. A.K.Bit, M.P.Biswal and S.S.Alam, Fuzzy programming approach to multiobjective solid transportation problem, Fuzzy Sets and Systems, 57(2) (1993) 183-194.

5. C.B.Cheng, Group opinion aggregationbased on a grading process: A method for constructing triangular fuzzy numbers, Computers and Mathematics with Applications, 48 (2004) 1619-1632.

6. S.Coupland, Type-2 fuzzy sets: Geometric defuzzification and type reduction, in Proceedings of the IEEE Symposium on Foundations of Computational Intelligence, Honolulu, HI (2007) 622-529.

7. D. Dubois and H. Prade, Fuzzy sets and systems: Theory and Applications, Academic Press, New York, 1980.

8. S.Greenfield, F.Chiclana, R.I.John and S.Coupland, The sampling method of defuzzification for type-2 fuzzy sets: Experimental evaluation, Information Sciences, 189 (2012) 77-92.

9. T.Hasuike and H.Ishi, A type-2 fuzzy portfolio selection problem considering possibilistic measure and crisp possibilistic mean value, IFSA-EUSFLAT 2009, 1120-1125.

10. F.L.Hitchcock, The distribution of a product from several sources to numerous localities, Journal of Mathematics and Physics 20 (1941) 224-230.

11. T.C.Koopmans, ed. Activity analysis of production and allocation. No. 13. New York: Wiley, 1951.

12. U.S.Palekar, M.K.Karwan and S.Zionts, A branch and bound method for the fixed charge transportation problem, Management Science, 36 (1990) 1092-1105.

13. P.Kundu, S.Kar and M.Maiti, Multi-objective multi-item solid transportation problem in fuzzy environment, Applied Mathematical Modelling, 37 (2013) 20282038.

14. C.S.Ramakrishnas, An improvement to Goyal's modiffed VAM for the unbalanced transportation problem, Journal of the Operational Research Society, 39 (1988) 609610.

15. M.Mizumoto and K.Tanaka, Fuzzy sets of type-2 under algebraic product and algebraic sum, Fuzzy Sets and Systems, 5(3) (1981) 277-280. 
Profit Maximization Solid Transportation Problem with Gaussian Type-2 Fuzzy Environments

16. E.Shell, Distribution of a product by several properties, Directorate of Management Analysis, Proceedings of the Second Symposium in Linear Programming, Vol. 2. 1955.

17. K.B.Haley, New methods in mathematical programming-The solid transportation problem, Operations Research, 10 (1962) 448-463.

18. A.Khanra, M.Maiti, T.Pal and M.Maiti, Special TSPs considering conveyances and routes through a hybrid algorithm, Annals of Pure and Applied MAthematics, 16 (2) (2018) 265-281.

19. N.Chakraborty, S.Mondal and M.Maiti, An EPQ model for deteriorating items under random planning horizon with some linguistic relations between demand, selling price and trade credit, ordered quality, Journal of Mathematics and Informatics, 6 (2016) 73-92.

20. P.Giri, M.Maiti and M.Maiti, Solid transportation problem under budget constraint using fuzzy measure, Annals of Pure and Applied Mathematics, 7(1) (2014) 41-46. 